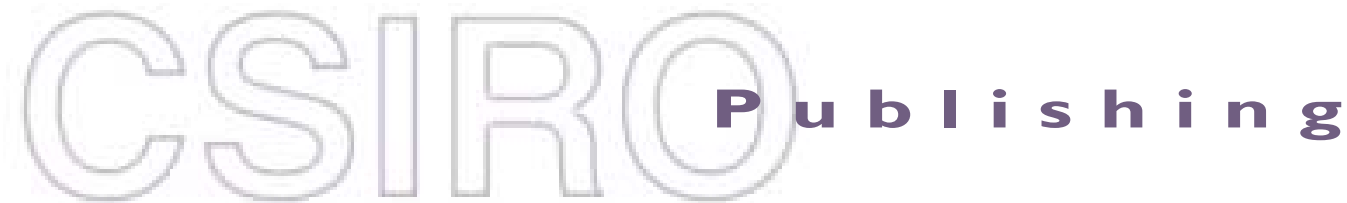

\section{Publications of the Astronomical Society of Australia}

Volume 19, 2002

(C) Astronomical Society of Australia 2002

An international journal of astronomy and astrophysics

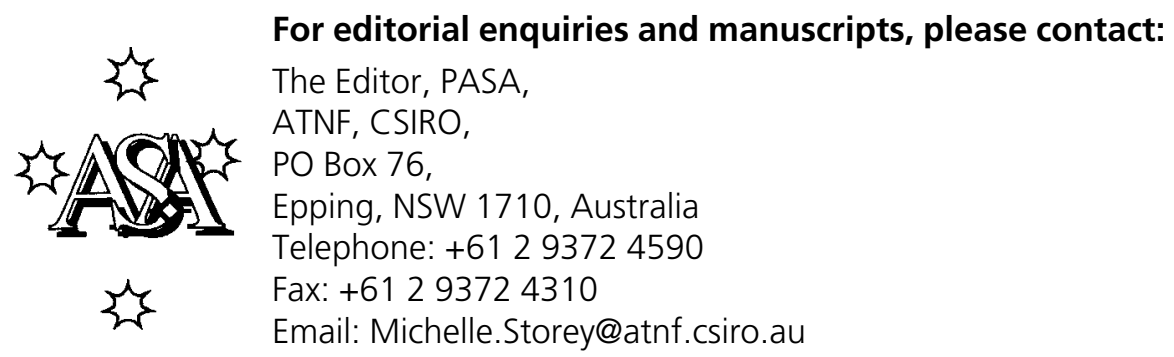

For general enquiries and subscriptions, please contact: CSIRO Publishing PO Box 1139 (150 Oxford St)

Collingwood, Vic. 3066, Australia

Telephone: +6139662 7666

Fax: +61 396627555

Email: publishing.pasa@csiro.au

C S I RO

PUBLISHING Published by CSIRO Publishing

for the Astronomical Society of Australia

www.publish.csiro.au/journals/pasa 


\title{
The 2001 Australian Galaxy Cluster Workshop
}

\author{
Michael J. Drinkwater* \\ School of Physics, University of Melbourne, Victoria 3010, Australia \\ Received 2001 October 19, accepted 2002 March 11
}

\begin{abstract}
The first 'Australian Cluster Workshop' was held at the Australia Telescope National Facility in Sydney on 2001 February 6. The aim of the workshop was to bring together the many and varied groups working on clusters of galaxies in Australia, to forge new multi-disciplinary links, and to generate enthusiasm and support for new cluster work and further cluster meetings in Australia. In this paper I present a summary of the workshop as well as some additional review material intended to place current Australian research in a broader perspective, looking ahead to the major issues still to be addressed.
\end{abstract}

Keywords: galaxies: clusters: general - cooling flows - intergalactic medium - cosmic microwave background

\section{Introduction}

In the last twenty or more years our understanding of galaxy clusters has changed dramatically. They were originally observed and classified as relatively stable collections of evolved galaxies, but even early on Zwicky (1937) showed that the reality was much more complex with his measurement of very high mass-to-light ratios in Coma. We now recognise galaxy clusters as the sites of intense activity due to a variety of physical processes, and that even the galaxies themselves are small players as they represent at most 10 per cent of the total cluster mass. Clusters are now also recognised as a major tool in the study of cosmology.

Australian astronomers have made a substantial contribution to these developments, notably through the long history of excellent wide-field spectroscopy on the AngloAustralian Telescope (AAT). The Butcher-Oemler effect was only really confirmed when the faint blue galaxies were spectroscopically identified by AAT observations (see Section 3.2 below). Nor should we forget the major role of the UK Schmidt Telescope (UKST) whose Southern Sky Survey formed the basis of the southern extension of Abell's cluster catalogue (Abell, Corwin, \& Olowin 1989, hereafter ACO).

The contribution of Australian observations to cluster science is even more important today with the increasing use of the wide field multi-object spectrographs of the Anglo-Australian Observatory (AAO) and the radio imaging capabilities of the Australia Telescope. Some of the recent results are described below, but major advances in the near future can also be anticipated as we start to analyse results from the recently completed HI Parkes All Sky Survey (HIPASS: Barnes et al. 2001) and the nearly completed 2dF Galaxy Redshift Survey (Colless 2001). Future initiatives in which Australia is involved include the new $6 \mathrm{dF}$ infrared-selected Galaxy Survey on the UKST (Saunders et al. 2001), and the AMiBA microwave array

\footnotetext{
* Now at: Department of Physics, University of Queensland, Queensland 4072, Australia. Email mjd@physics.uq.edu.au
}

(Lo et al. 2000) for which the Australia Telescope National Facility (ATNF) is building receivers.

International interest in cluster astronomy has been stimulated by the many excellent results now appearing from the new XMM-Newton and Chandra satellites. These $\mathrm{X}$-ray satellites have led to the revision of several aspects of cluster science as described below. Many of these results were presented at recent international meetings such as the 2000 Institut d'Astrophysique de Paris meeting 'Constructing the Universe with Clusters of Galaxies' (Durret \& Gerbal 2000) and the 2001 XXIth Moriond Astrophysics Meeting 'Galaxy Clusters and the High Redshift Universe Observed in X-Rays' (proceedings to be published electronically).

Interest in these many aspects of cluster astronomy led to the holding of the first 'Australian Cluster Workshop' held in Sydney on 2001 February 6 at the ATNF. The main purpose of the meeting was to bring together all those working on cluster-related science in Australia to share news of their latest work, but it was also an opportunity to discuss plans for a proposed cluster symposium for the Sydney 2003 IAU General Assembly. The aim of this paper is to give a summary of the workshop as well as a brief introduction to the field. The review material is mostly limited to material presented at the workshop; for a more general introductory review see Carlberg (2001). I have included some of the review material in Section 2, a brief introduction to the physics of clusters. This is followed by the main account of topics discussed at the workshop which I have split into Section 3, the evolution of cluster galaxies and Section 4, the properties of the intra-cluster medium. In Sections 3 and 4, all the references are to work by participants of the workshop, except for those given as footnotes or specifically identified as review material.

\section{Cluster Physics}

The aim of this section is to review some of the basic physical properties of galaxy clusters as a whole. Smaller-scale processes acting on individual components of clusters 
are reviewed where necessary in the later sections. Some readers will want to skip this section and go directly to the main discussion starting in Section 3.

\subsection{Observational Definition of Clusters}

Historically clusters have been defined entirely on the basis of prominent concentrations (over-densities) in the distribution of optical galaxies. The definitive compilation by Abell (1958) defined galaxy concentrations as clusters if they had more than 50 galaxies within a $2 \mathrm{Mpc}\left(H_{0}=\right.$ $75 \mathrm{~km} \mathrm{~s}^{-1} \mathrm{Mpc}^{-1}$ ) radius and no more than 2 magnitudes fainter than the third brightest member. With this definition and including the southern extension (ACO), the final catalogue contains about 4000 clusters to a redshift of $z=0.25$. This remains the most common definition despite the recognition that galaxies only contribute a small fraction of the total masses of clusters. More recently the large fraction of rich clusters with strong X-ray emission has led to cluster samples defined entirely by the detection of extended X-ray emission (Ebeling et al. 2000, and references therein). Although the X-ray cluster samples are limited to relatively low redshifts, they avoid some of the uncertain selection effects present in optical samples and have led to important results for cluster physics and cosmology in general (for a brief review see Schindler 2001).

\subsection{Cluster Masses}

The dynamical estimation of cluster masses has a long history dating back to the pioneering work of Zwicky (1937) described above. The cluster masses are normally estimated by assuming they are dynamically relaxed and invoking the viral theorem (for example Colless \& Dunn 1996). A more general mass estimator has been proposed by Diaferio (1999) which identifies caustic-like structures in the velocity distribution of cluster galaxies and does not make the usual assumption of dynamical equilibrium. Australian multi-object spectrographs are ideal cluster redshift machines and have contributed to measurements of many more clusters (from Couch \& Sharples 1987, to Drinkwater, Gregg, \& Colless 2001a), but Zwicky's basic result still holds. Galaxy clusters have very high mass-tolight ratios, typically around 300 in solar units (Bahcall et al. 2000).

Measurements of the hot X-ray gas in clusters can also be used to derive their total gravitational masses. The gas can be assumed to be in hydrostatic equilibrium, so the gravitational potential is directly related to the gas density and pressure, both of which can be inferred from the $\mathrm{X}$-ray measurements. X-ray cluster masses are generally consistent with dynamical estimates, but substructure can bias the X-ray estimates (e.g. Machacek et al. 2001) and in poor clusters they are limited to the central regions where gas is detected (e.g. Ikebe et al. 1996). For an overview of the hydrostatic and dynamical techniques, see Tremaine (1992).

Finally, gravitational lensing is a particularly powerful method of measuring cluster masses. The distortion of background images by a cluster is directly related to the projected mass distribution. In the cores of rich clusters, the mass density can be high enough to form multiple arc-like images as shown in the spectacular Hubble Space Telescope image of Abell 2218 (Kneib et al. 1996) in Figure 1. This 'strong' lensing allows for very detailed mapping of the mass distribution in the central few hundred $\mathrm{kpc}$ of clusters. At larger projected radii the smaller mass density results in weaker gravitational distortions ('weak lensing') which can be analysed statistically to infer the mass distribution, although with greater uncertainties (e.g. Clowe \& Schneider 2001). Since gravitational lensing traces the total mass distribution, it potentially allows us to measure the distribution of the dark matter in clusters (e.g. Tyson 1996).

These three different methods give generally consistent estimates of cluster masses. The total mass of a typical rich cluster is around $10^{15} \mathrm{M}_{\odot}$, of which galaxies make up at most $4 \%$, the hot gas around $17 \%$, and the remainder is dark matter (Ettori \& Fabian 1999).

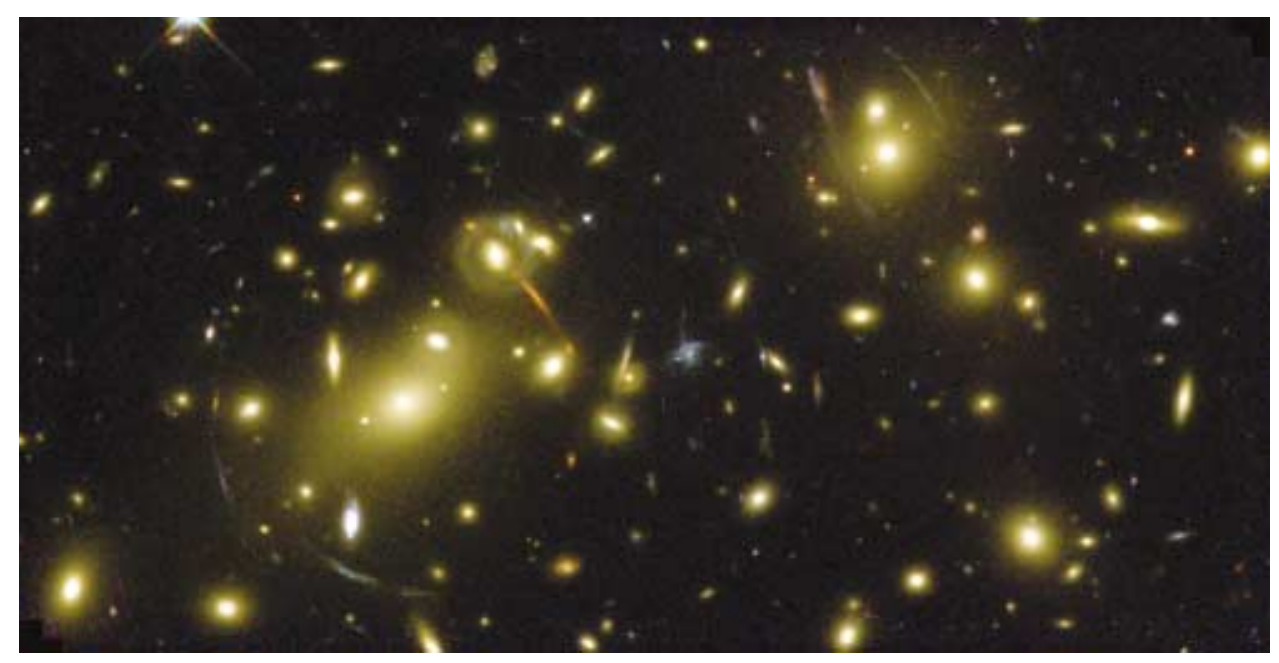

Figure 1 Optical image of cluster Abell 2218 taken with the WFPC2 camera of the Hubble Space Telescope by Kneib et al. (1996). (W. Couch) 


\subsection{Cluster Formation}

Any real discussion of the theoretical basis of cluster formation is well beyond the scope of this current paper, so I shall just mention a few of the problems and challenges currently of interest. Clusters are the largest structures to have broken away from the Hubble expansion as a result of their own gravity. The distance at which this occurs (the 'turn-around' radius) is, in principle, directly related to the Hubble constant and the matter density of the universe (Praton \& Schneider 1994). In practice, however it has proved hard to measure this well enough to derive cosmological parameters, although the method of Diaferio (1999) mentioned above can provide robust mass estimates.

On smaller scales, hierarchical models of galaxy formation are very successful at describing the formation of galaxies within massive cluster-like halos of dark matter. However these generally predict many more low-mass systems than are actually observed (e.g. White \& Frenk 1991). It is not yet clear if the discrepancy is caused by merging of the smaller systems, or an observational bias against detecting the low-mass systems. For this reason it is especially important to obtain better measurement of the faint end of the cluster galaxy luminosity function (see Section 3.1). More recent work suggests that high-density clusters do have the expected numbers of low-mass systems and that the deficiency in low-density regions is due to their formation being suppressed by photoionisation of the intergalactic medium at the later epochs when these regions formed (e.g. Tully et al. 2001).

The timescales for cluster infall can be very long so we should not assume the formation is complete: many clusters show clear evidence of subpopulations of galaxies being currently accreted (e.g. Colless \& Dunn 1996). Although typical crossing times are around $6 \times 10^{8} \mathrm{yr}$ for a diameter of $1 \mathrm{Mpc}$ and a radial velocity dispersion of $1000 \mathrm{~km} \mathrm{~s}^{-1}$, the collapse time ${ }^{1}$ is of order $10^{9} \mathrm{yr} \times$ $\left[(R / \mathrm{Mpc})^{3} /\left(M / 10^{15} \mathrm{M}_{\odot}\right)\right]^{1 / 2}$ which can be more than a Hubble time, depending on the cluster mass. Longer still (although it depends on the galaxy mass) is the relaxation time $^{2}$ for galaxies within the cluster, of order $2 \times 10^{12} \mathrm{yr}$.

\section{The Evolution of Cluster Galaxies}

It has long been recognised that clusters are ideal laboratories in which to study galaxy populations, especially the smaller dwarf galaxies which are very hard to detect let alone study outside these high-density regions. Historically, attention has focused on what clusters can tell us about the global properties of their galaxy populations such as the luminosity function. More recently the focus has moved towards what cluster galaxies can tell us about the formation and subsequent evolution of galaxies, especially now that observations of high-redshift clusters show

\footnotetext{
${ }^{1}$ Binney \& Tremaine (1987), p 38.

${ }^{2}$ Binney \& Tremaine (1987), p 514.
}

changes with cosmic epoch. This new emphasis on the processes at work in clusters dominated the work presented at the workshop, but I shall start by reviewing our current understanding of the galaxy populations we do find in clusters today. Note that we must remember the caveat that galaxy clusters are a special environment where galaxies are strongly affected by the local conditions. For excellent reviews of galaxy evolution in clusters see Dressler (1984) and (in the radio context) van Gorkom (1996).

\subsection{Galaxy Populations in Clusters}

Nearby galaxy clusters such as Virgo, Fornax, and Coma have dominated our understanding of galaxy populations - especially dwarfs - over the last two decades. The definitive work based on photographic surveys by Sandage and collaborators (Virgo) and Ferguson $\&$ Sandage (Fornax) led to the first descriptions of type-specific luminosity functions ${ }^{3}$. The total luminosity function was found to be made up of Gaussian-like distributions of early and late type giant galaxies at high luminosity, plus a flat or even rising distribution of dwarf ellipticals, the dominant population at low luminosities. These surveys relied on very high quality photographic plates used to classify which galaxies were cluster members based on morphological criteria. These classifications have proved very reliable (Drinkwater \& Gregg 1998), but there was always a concern that the samples were biased by preconceptions of what cluster galaxies should look like.

The advent of efficient wide-field multi-object spectrographs has led to a second generation of cluster studies where visual classifications have been replaced by objective redshift measurements to determine which galaxies are true cluster members. Extensive cluster studies have been made in Australia thanks to the AAO's expertise in multi-object optical fibre spectrographs. These include the 97-fibre FLAIR-II system of the UKST (now replaced by the 150 -fibre $6 \mathrm{dF}$ ) and the 400 -fibre $2 \mathrm{dF}$ on the AAT (Lewis et al. 2001). The spectroscopic observations do much more than just confirm cluster memberships: the velocities reveal the internal dynamics of clusters (see Section 3.4) and the spectral features reveal the internal composition and activity of the individual galaxies, such as star formation discussed in Section 3.3.

The huge multiplex advantage of the multi-object spectrographs has made it possible to search for unusual cluster galaxies among the objects that were previously ignored because they did not look like cluster members. Drinkwater \& Gregg (1998) used the FLAIR-II system to observe some 500 galaxies of compact morphology in the direction of the Fornax Cluster. They found that nine of these were actually members of the cluster small, normal surface brightness dwarfs that had been misclassified as background galaxies in the earlier morphological studies. On the basis of this evidence of a bias

\footnotetext{
${ }^{3}$ Binggeli, Sandage, \& Tammann (1988); Ferguson \& Sandage (1988).
} 
in existing galaxy samples, they extended this approach in the Fornax Cluster Spectroscopic Survey by using the $2 \mathrm{dF}$ spectrograph to measure spectra of all objects in the central region of the cluster (brighter than $b_{j}=19.7$ ), both 'stars' and 'galaxies', to avoid any bias against compact galaxies (Drinkwater et al. 2000a). This approach was rewarded by their discovery of an entirely new population of 'ultra-compact' dwarf (UCD) galaxies in the cluster (Drinkwater et al. 2000b). The UCDs are mostly unresolved in ground-based imaging and have spectra like old stellar systems; they may be massive intra-cluster globular clusters or they may be the cores of nucleated dwarfs that have been tidally stripped. Recent numerical simulations of this process, described as 'galaxy threshing' (Bekki, Couch, \& Drinkwater 2001a), have shown that dwarfs that pass close to the central galaxy NGC 1399 can be disrupted in this way.

Despite these advances in spectroscopic cluster surveys, most measurements of cluster galaxy luminosity functions (LFs) are still based on imaging surveys, and there is still no agreement over the numbers of low luminosity galaxies. There have been many reports of large numbers of faint galaxies in clusters characterised by a luminosity function with a steep slope at the faint end (references to several are given by Boyce et al. 2001). In the extreme case, a very steep LF could entail the dwarf galaxies making up a significant fraction of the total cluster mass. Steep luminosity functions are more consistent with the predictions of hierarchical galaxy formation, but the measurements of steep LFs are not universally accepted as they are all based on statistical arguments to correct for background galaxies. Recently R. De Propris et al. (in preparation) have analysed a cluster sample from the 2dF Galaxy Redshift Survey with complete spectroscopic identification and drawn two major conclusions. Firstly, for brighter galaxies $\left(M_{B}<-16\right)$ the cluster LF is the same as the field LF apart from a normalisation: this is a problem for the cold dark matter model of galaxy formation as it predicts the mass distribution in clusters should be very different to that of field galaxies ${ }^{4}$. Secondly, they show with full spectroscopic identifications that the background subtraction methods are reliable over their range of magnitudes $\left(b_{j}<19.45 \mathrm{mag}\right)$, supporting the previous statistical estimates.

Another observational window on clusters is HI radio emission from neutral hydrogen. The HIPASS survey gives $\mathrm{HI}$ measurements across the whole southern sky, but is limited to the more massive galaxies: $M_{\mathrm{HI}}>10^{8} \mathrm{M}_{\odot}$ at the distances of typical nearby clusters. Waugh, Webster, \& Drinkwater (2000) used the HIPASS data to study the distribution of neutral hydrogen in a very large region (100 square degrees) around the Fornax Cluster. The HI distribution, shown in Figure 2, does not show the extreme overdensity seen in optical galaxies in the cluster core region $^{5}$ (core radius $240 \mathrm{kpc}$ ). The cluster is nevertheless

\footnotetext{
${ }^{4}$ Dekel \& Silk (1986).

${ }^{5}$ Ferguson (1989).
}

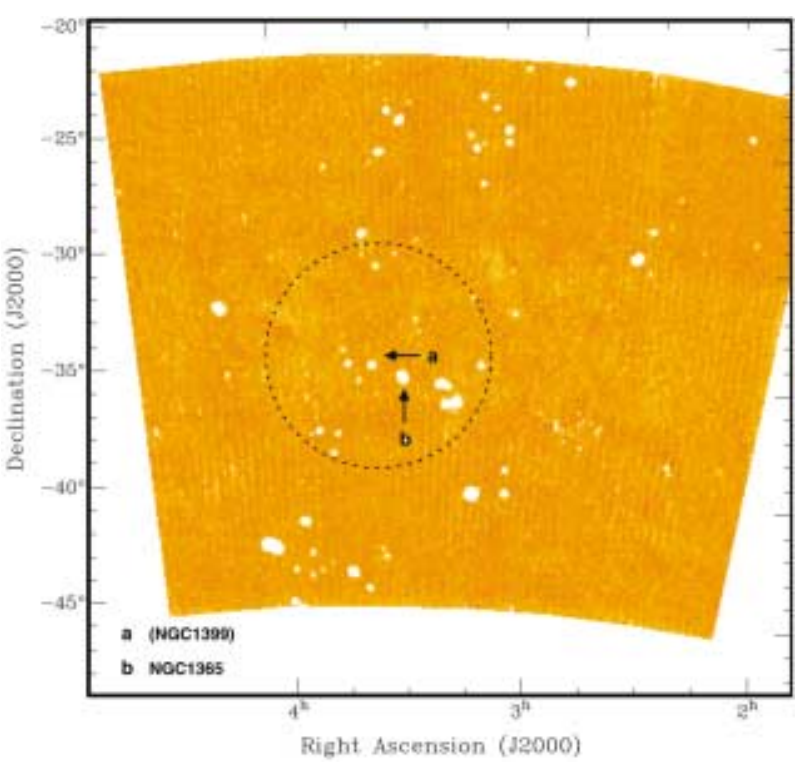

Figure 2 HI image of Fornax Cluster: a mosaic of HIPASS data cubes showing the Fornax region in $\mathrm{HI}$ over the range $500-2500 \mathrm{~km} \mathrm{~s}^{-1}$. The elliptical central galaxy of the cluster, NGC 1399, is not detected in HI, whereas several bright spirals including NGC 1365 (indicated) are clearly detected. The dotted circle at a radius of $5^{\circ}(1.75 \mathrm{Mpc})$ from NGC 1399 indicates the approximate extent of the Fornax Cluster as detected optically. (M. Waugh)

detected as an increase in the surface density of $\mathrm{HI}$ selected galaxies, but at a lower level on larger scales: an overdensity of $3 \pm 1$ within a 4 degree $(1.4 \mathrm{Mpc})$ radius of the cluster centre. Neutral hydrogen does not survive in the central region of clusters but can be detected in the outer regions: presumably these are galaxies still falling into the clusters which have not yet been stripped of their gas. Waugh et al. (2000) also found that the cluster is part of a very large sheet of galaxies extending beyond the $10 \mathrm{Mpc}$ extent of their survey.

\subsection{Morphological Transformation in the Past: the Butcher-Oemler Effect}

Looking at more distant clusters there is a dramatic change in the galaxy populations. The fraction of blue galaxies increases rapidly from the current epoch out to a redshift of $z=0.6$ or more: the Butcher-Oemler effect ${ }^{6}$. This change was first identified as an excess of blue galaxies in images of distant cluster cores. AAT observations were among the first to confirm that these blue galaxies were indeed cluster members, by narrow-band photometry (Couch et al. 1983) and spectroscopy (Couch \& Sharples 1987). More recently, Hubble Space Telescope imaging has confirmed that the blue 'Butcher-Oemler' galaxies are dominated by spiral types (Couch et al. 1998, and references therein). In the infall scenario described above, the Butcher-Oemler effect would arise if the infall rate were higher in the past and the cluster environment were less hostile so that star

\footnotetext{
${ }^{6}$ Butcher \& Oemler (1978).
} 


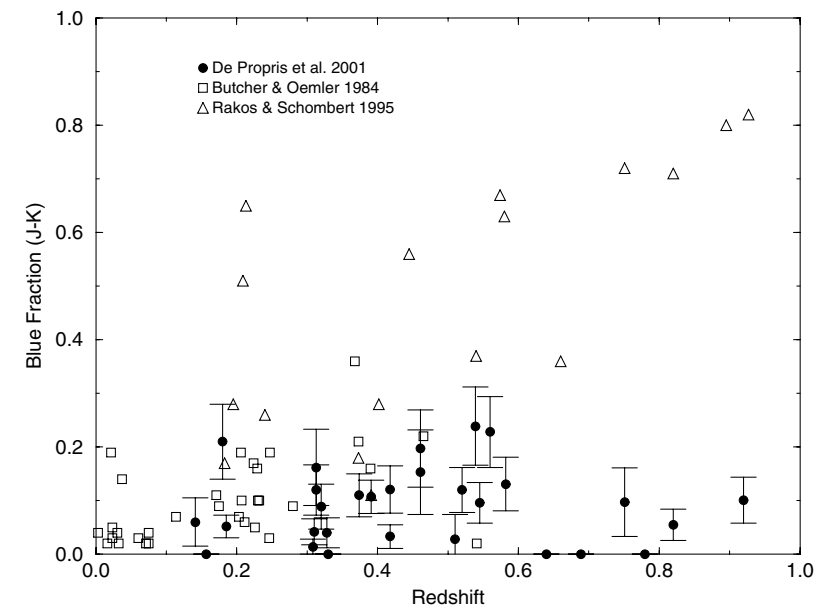

Figure 3 Butcher-Oemler effect: The blue fraction (the number ratio of galaxies bluer than 0.2 magnitudes in $B-V$, transformed to $J-K$ following Smail et al. 1998) in a $0.7 \mathrm{Mpc}$ (radius) region centred on the brightest cluster galaxy, as a function of cluster redshift (black dots: R. De Propris et al., in preparation). Open squares and triangles are previous determinations of blue fractions which show a strong Butcher-Oemler effect compared to the infrared-selected galaxies. (R. De Propris)

formation was not halted immediately a galaxy entered the cluster (Couch, this workshop).

The situation is quite different when infrared-selected cluster galaxy samples are used: this selects older stellar populations than the optical samples which are mostly dominated by young blue stellar populations. In a K-band selected sample of galaxies in 38 clusters R. De Propris et al. (in preparation) find that the fraction of blue galaxies is much smaller than in the earlier optical samples; see Figure 3 which also shows other measurements of the effect. Furthermore they find no significant variation in the blue fraction with redshift. They therefore argue that a large part of the observed Butcher-Oemler effect is due to star-bursting dwarf galaxies which, although a small part of the galaxy population as measured by mass, are included in the optical samples because of their blue colours.

Finally, extremely high redshift $(z>2)$ clusters can be located by the detection of multiple, correlated Ly $\alpha$ absorption systems in QSO spectra. Francis, Wilson, $\&$ Woodgate (2001) have identified a cluster (2142-4420) at a redshift of $z=2.4$ in this way, although they note that the system is unlikely to be gravitationally bound and may not satisfy the normal definition of a cluster. The 'cluster' consists of a total of seven absorption systems in three QSO spectra plus three very strong Ly $\alpha$ emitting galaxies. Their analysis shows that this cluster (and perhaps all high redshift clusters) is surrounded by a $5 \mathrm{Mpc}$ structure of neutral hydrogen of total mass $10^{12} \mathrm{M}_{\odot}$. They infer that this gas must either collapse into stellar systems or become ionised, forming the hot intra-cluster medium seen at the current epoch. Are we seeing clusters in the process of formation?

\subsection{Morphological Transformation: Halting Star Formation}

In the early photographic cluster surveys the galaxies were given morphological classifications along the traditional Hubble sequence supplemented by dwarf classifications. For both giants and dwarfs the 'late-type' classification indicated gas-rich galaxies with significant populations of young stars ${ }^{7}$. A striking property of the galaxy populations in rich clusters is the very high ratio of early-type to late-type galaxies compared to the field galaxy population. This is known as the density-morphology relation ${ }^{8}$. Spectroscopic measurements of large numbers of cluster galaxies now make it possible to augment the morphological classifications with direct measurements of both current and recent star formation rates (from optical emission lines and Balmer absorption lines respectively). In the Coma Cluster Poggianti et al. (2001) used spectroscopic analysis of a large sample of elliptical and S0 galaxies to determine the ages of their stellar populations. They found that $40 \%$ of the S0s had recently (within 5 Gyr) undergone star formation in their central regions compared to none of the ellipticals. Interestingly they found that this recent star formation activity was more common in the less luminous S0s $\left(M_{B}>-19\right)$. This is consistent with dynamical observations that dwarf cluster galaxies represent an infalling population which may not yet have had star formation suppressed by the cluster environment (see Section 3.4).

There is a general agreement between the morphological and spectroscopic classifications but there are notable exceptions, especially in more distant clusters. Poggianti et al. (1999) found that many galaxies morphologically classified as spirals in high redshift $(z \approx 0.4)$ clusters have spectra indicating suppressed rates of star formation. They concluded that galaxy evolution in clusters can be separated into morphological and spectral evolution driven by different processes or on different timescales.

The raw material for star formation, neutral hydrogen, can also be measured at radio frequencies by HI emission as discussed above. Optical and HI spectroscopy have been used to study star formation in the Fornax Cluster by Drinkwater et al. (2001b) and Schröder, Drinkwater, \& Richter (2001) respectively. The optical data reveal a much higher rate of star formation among the dwarf galaxies than suggested by their morphological classification. The star formation rate is very low in the dwarf galaxies at the centre of the cluster, but is much higher in the infalling subcluster, presumably as it still contains significant amounts of HI gas. The radio HI data show that the cluster galaxies of a given type contain significantly less HI gas than field galaxies of the same type. For some of the dwarf galaxies, HI detections also allow the 'gas depletion' timescales to be estimated. The dwarfs at the edge of the cluster have short-lived bursts whereas those closer to the centre could sustain the current SFRs for several Gyr.

\footnotetext{
${ }^{7}$ A summary of the classification scheme can be found in de Vaucouleurs et al. (1991).

${ }^{8}$ Dressler (1980).
} 


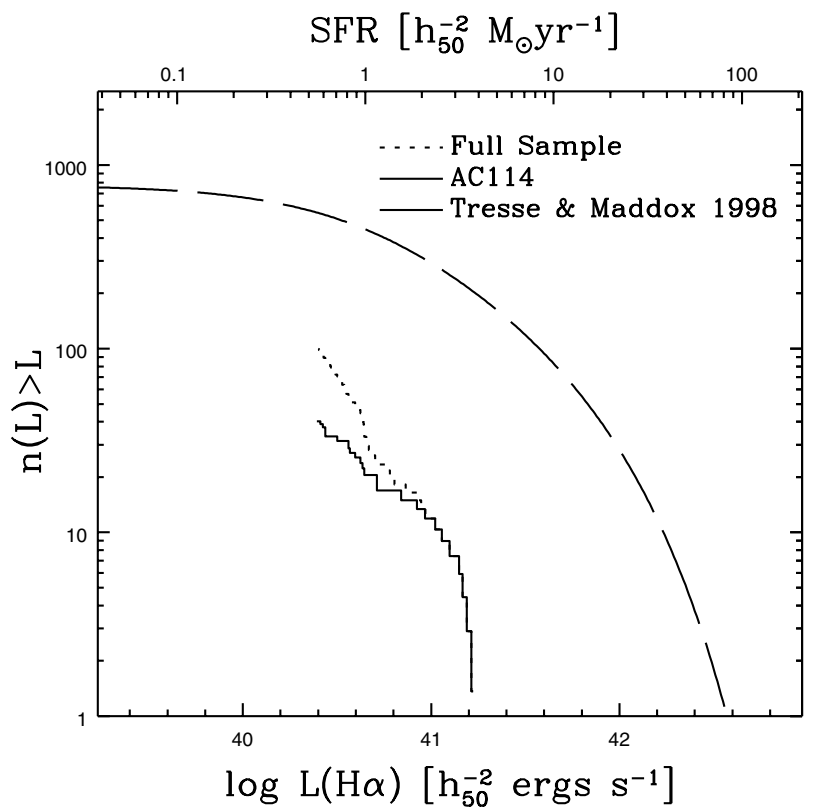

Figure 4 A comparison of the $\mathrm{H} \alpha$ luminosity function measured for AC 114 with that of field galaxies at a similar redshift (dashed line) measured by Tresse \& Maddox (1998). The corresponding star formation rate is shown on the top axis. (W. Couch)

There is further evidence for the suppression of star formation in more distant (but still 'low redshift') clusters. These intermediate redshift clusters contain galaxies with spectra indicative of recently interrupted star formation (the 'E+A' galaxies; see Poggianti et al. 1999). Recent direct measurements of star formation in the cluster AC114 at a redshift of $z=0.32$ have used the LDSS ++ spectrograph on the AAT to search for $\mathrm{H} \alpha$ emission lines in the spectra of nearly 700 cluster galaxies (Couch et al. 2001). The luminosity function of $\mathrm{H} \alpha$ emission in the cluster is an order of magnitude lower than that of a sample of field galaxies at the same redshift; see Figure 4. This suppression of star formation by the cluster environment extended over the whole range of distance from the cluster centre measured $(0-2 \mathrm{Mpc})$. This surprising result shows that the mechanism(s) responsible for suppressing star formation in clusters are effective well beyond the radii at which ram pressure stripping is expected to be significant.

The spectroscopic observations described above not only confirm the density-morphology relation, but indicate possible mechanisms for its origin. The densitymorphology relation arises naturally if star forming galaxies accreted onto clusters experience strong interactions resulting in a truncation of star formation and morphological change into early types. The formation of the ' $E+A$ ' galaxies seen in clusters has been demonstrated in numerical simulations by Bekki, Shioya, \& Couch (2001b). They show that these galaxies can result from the dynamical evolution of a dusty starburst associated with a major galaxy merger. An ambitious way of investigating such processes observationally is to make very sensitive $\mathrm{HI}$ observations of $z \approx 0.2$ clusters to directly observe morphological transformation of the infalling galaxies. The

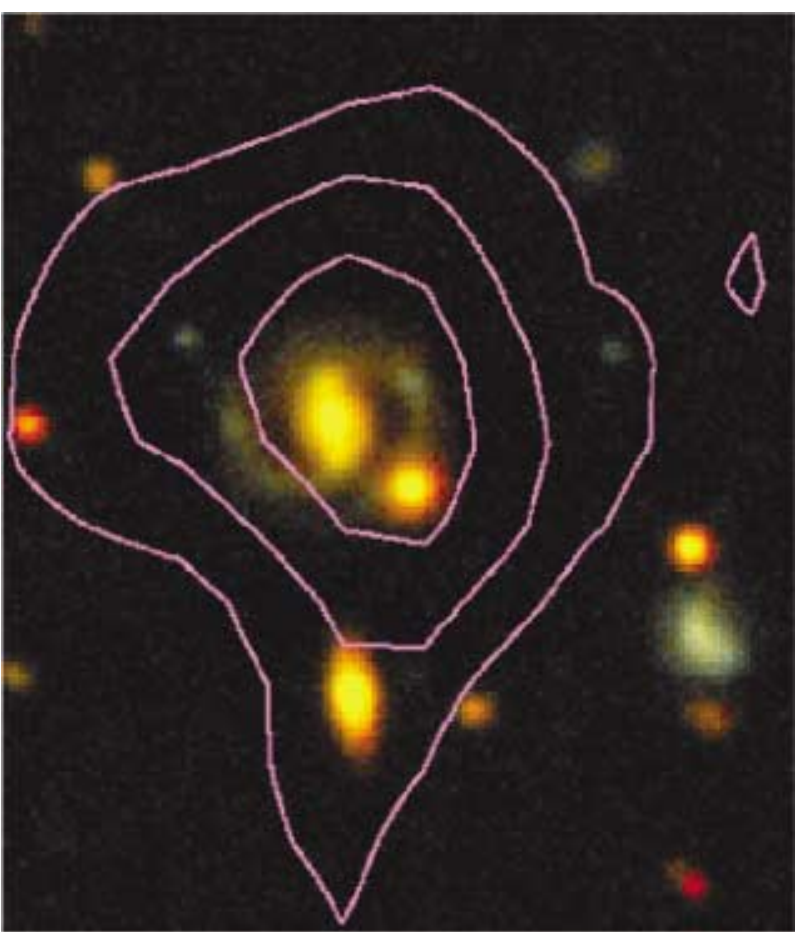

Figure 5 Detection of $\mathrm{HI}$ in the cluster Abell 2218. The HI measurements are shown as contours overlaid on a colour representation of the optical image. The HI data are from the Westerbork Telescope whose synthesised beam is $18.0^{\prime \prime} \times 19.7^{\prime \prime}$ in size, comparable to the extent of the second contour. The optical imaging observations were obtained on the W.M. Keck II Telescope. The size of the image is $60^{\prime \prime} \times 60^{\prime \prime}$ which corresponds to $184 \times 184 \mathrm{~h}_{65}^{-1} \mathrm{kpc}$ at the redshift of Abell 2218. (M. Zwaan)

feasibility of detecting $\mathrm{HI}$ at these distances has been demonstrated by Zwaan, van Dokkum, \& Verheijen (2001) in deep observations of the redshift $z=0.18$ cluster A 2218 as shown in Figure 5. They identified their HI detection with a spiral galaxy some $2 \mathrm{Mpc}$ from the cluster centre on the basis of the position agreement and a very similar redshift measured with the Keck I telescope. Although this work demonstrates that $\mathrm{HI}$ can be detected in more distant clusters, the spatial resolution will make it hard to draw any conclusions about the details of any morphological transformation taking place. A better strategy might be to compare the total $\mathrm{HI}$ content of distant and nearby clusters to see if this shows any significant evolution.

\subsection{Cluster Dynamics}

Multi-object spectroscopy has made possible detailed dynamical analyses of clusters using samples of hundreds of galaxies with measured redshifts. These studies not only confirm the high masses alluded to above, but they also reveal significant substructure, as do X-ray observations $^{9}$. Both Coma (Colless \& Dunn 1994) and Fornax (Drinkwater et al. 2001a) have significant infalling subclusters. It may turn out that most clusters have dynamically evolving substructure, even if their subclusters can be classified as relaxed. These subclusters are infalling

\footnotetext{
${ }^{9}$ Zabludoff \& Zaritsky (1995).
} 
and will merge with their parent clusters. As a result of this merging, very large amounts of kinetic energy can be injected into the intra-cluster medium: this is discussed below.

The dynamical studies also show that cluster dwarf galaxies have a higher velocity dispersion than the giants and may represent a newer infalling population (e.g. the Fornax Cluster: Drinkwater, Gregg, \& Colless 2001a ${ }^{10}$. In contrast, measurements of the more distant Coma Cluster have not yet detected any significant difference in the dynamics of its dwarf galaxy population (Edwards \& Colless 2001).

The radio structures of many cluster galaxies show strange features thought to result from dynamical interactions with the intra-cluster medium (ICM). Such interactions range from distortions of the normal radio lobes seen in field galaxies to very unusual features seen only in clusters ('tailed' radio sources). There is still some debate as to whether they form as a result of the galaxies moving through the ICM or if they are caused by ICM 'weather' impacting on the galaxies. Cluster weather is the most accepted explanation for the highly curved 'narrow angle' tailed radio sources ${ }^{11}$ because they have average velocities within clusters and not the high velocities needed to form the tailed structures by motion through a calm ICM. In smaller clusters and groups however, such as the compact group, J0321-455, shown in Figure 6, motion of the galaxies themselves is the preferred explanation (Klamer, 2000; Hunstead, this workshop). A similar explanation of radio jets bent due to motion of a galaxy through the ICM in a poor cluster was found by Read et al. (2001) for the peculiar galaxy pair in the cluster Abell S0102.

\section{Processes in the Intra-cluster Medium}

As described above clusters are mostly composed of material other than galaxies: the as yet unidentified dark matter and the intra-cluster medium which is dominated by the hot gas observed in X-ray emission. In this section I describe work presented dealing with the many varied processes going on in the intra-cluster medium.

\subsection{Hot Intra-cluster Gas}

Galaxy clusters and groups contain enormous amounts of hot gas. The total mass of gas can exceed that of the galaxies making up the cluster and can reach $20 \%$ or more of the total mass of the cluster. This gas is revealed by thermal bremsstrahlung radiation at X-ray frequencies: it is very hot with temperatures of order $10^{8} \mathrm{~K}$. The X-ray observations also reveal substructure in many clusters, interpreted as evidence of merging at work, such as in the Coma Cluster shown in Figure 7. A major issue raised by early X-ray cluster measurements was the very high gas cooling rates inferred. The first generation of X-ray satellites showed that about half the clusters have bright X-ray emission

\footnotetext{
${ }^{10}$ See Conselice, Gallagher, \& Wyse (2001) for a discussion of similar results in the Virgo Cluster.

${ }^{11}$ See review by Burns (1998).
}

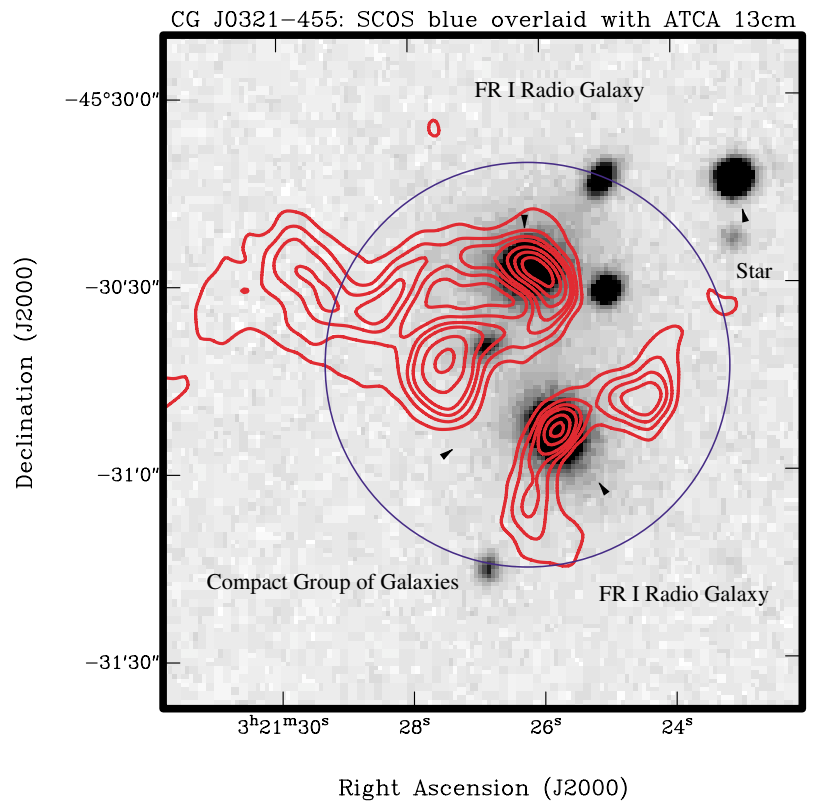

Figure 6 ATCA $13 \mathrm{~cm}$ contours overlaid on the SuperCOSMOS blue image of the extraordinary compact group CG J0321-455 at $z=0.07$. Contours are at $1,2,3,4,6,8,10 \mathrm{mJy} / \mathrm{beam}$ and the beam size is $5^{\prime \prime} \times 4^{\prime \prime}$. The extent of the compact group is marked by the blue circle, and the bright host galaxies of the two tailed FR I radio sources are arrowed. Its high integrated radio power $\left(P_{1.4}=5 \times 10^{24} \mathrm{~W} \mathrm{~Hz}^{-1}\right)$ and the presence of $t w o$ tailed sources make this compact group unique. (I. Klamer \& R. Hunstead)

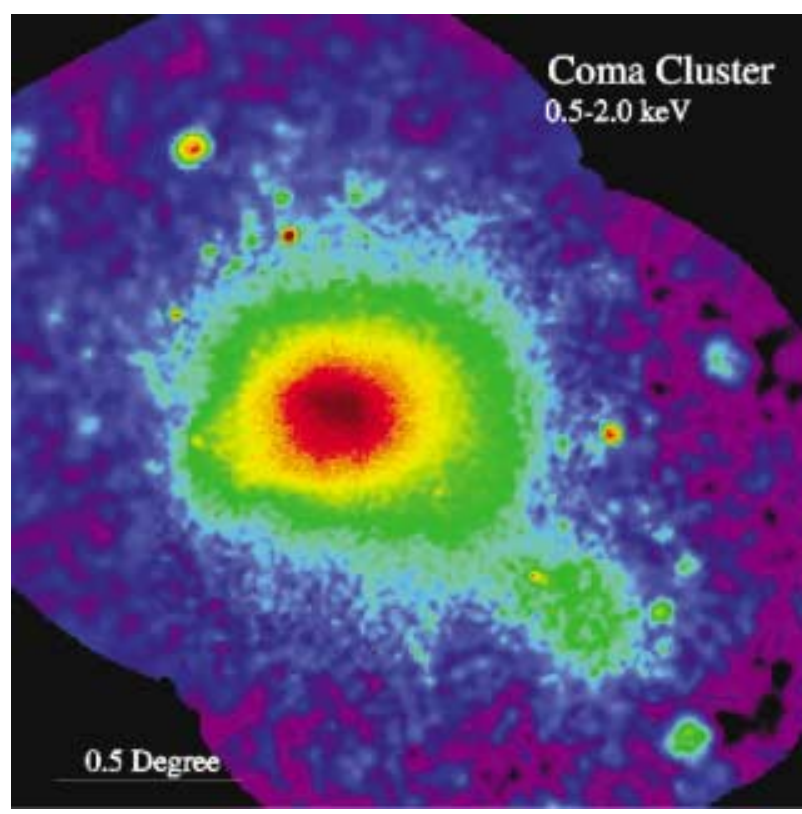

Figure 7 The Coma Cluster measured in X-rays with ROSAT.

in the cores associated with 'cooling flows' - dense gas with short radiative cooling times. The cooling gas was presumed to accrete on to the central galaxies to form giant molecular clouds associated with the observed star formation, but the observed star formation rates were too low to be consistent with the accretion rates from the cooling flows. For a review of the evidence for cooling flows 
and other references to X-ray observations of clusters see Fabian (1994).

The new X-ray missions (Chandra and XMM-Newton) have resolved these central regions with spectroscopic observations. Spectroscopic measurements of the cooling rates are 5-10 times less than the original estimates. This means there is no longer such a problem with what happens to the cold gas, but there are still significant amounts of cooling gas inferred. In some cases this is still greater than the measured star formation rates in the central galaxies, so there must be a heating mechanism to balance the cooling (McNamara et al. 2000). Detailed Chandra observations of Hydra A show 'holes' in the X-ray emission coincident with radio lobes of the central galaxy: see Figure 8. David et al. (2001) find that the central cooling flow region is convectively unstable because of heating by the central radio source, resulting in a reduced accretion rate. The energetics of the cluster gas will also be strongly affected by cluster mergers: during a merger a large fraction of the kinetic energy will be dissipated in the gas. Remarkably, the dense gas cores can survive well into the merger process as observed in the complex structure seen in Abell 2141 (Markevitch et al. 2000). A detailed analysis of the different heating and cooling mechanisms of the intracluster gas is given by Wu, Fabian, \& Nulsen (2000) who note the importance of non-gravitational processes such as the heating effect of active galactic nuclei (AGN) in the central galaxies.

The hot cluster gas can also be detected through its effect on background photons from the cosmic microwave background. These are scattered to higher energies by the gas, which is then detected as a shift - the SunyaevZel'dovich (SZ) effect - in the CMB spectrum localised to the projected position of the cluster. Although there were only a few positive detections of the SZ effect in the first two decades after it was proposed ${ }^{12}$, there have now been around 40 detections; see Carlstrom et al. (2000) for a full review of the current status of SZ science. SZ measurements of clusters are extremely important for cosmology because they can detect clusters with a sensitivity that is independent of distance, allowing the number of high redshift clusters to be measured, directly constraining cosmological parameters. Furthermore, the physical size of clusters can be measured by combining SZ-measured density profiles with independent X-ray measurements of the surface brightness and temperature profiles. The Array for Microwave Background Anisotropy (AMiBA; see Lo et al. 2000 ) is being built specifically to exploit the full potential of the SZ effect for high redshift cluster detections. There is direct Australian involvement in this project as the ATNF is supplying receiver technology. AMiBA is a ground-based instrument with 1 arc minute resolution at 85-105 GHz, expected to start operating early in 2004. It will be much more sensitive than X-ray satellites at detecting clusters at redshifts greater than $z=0.6$.

\footnotetext{
${ }^{12}$ Sunyaev \& Zel'dovich (1972).
}

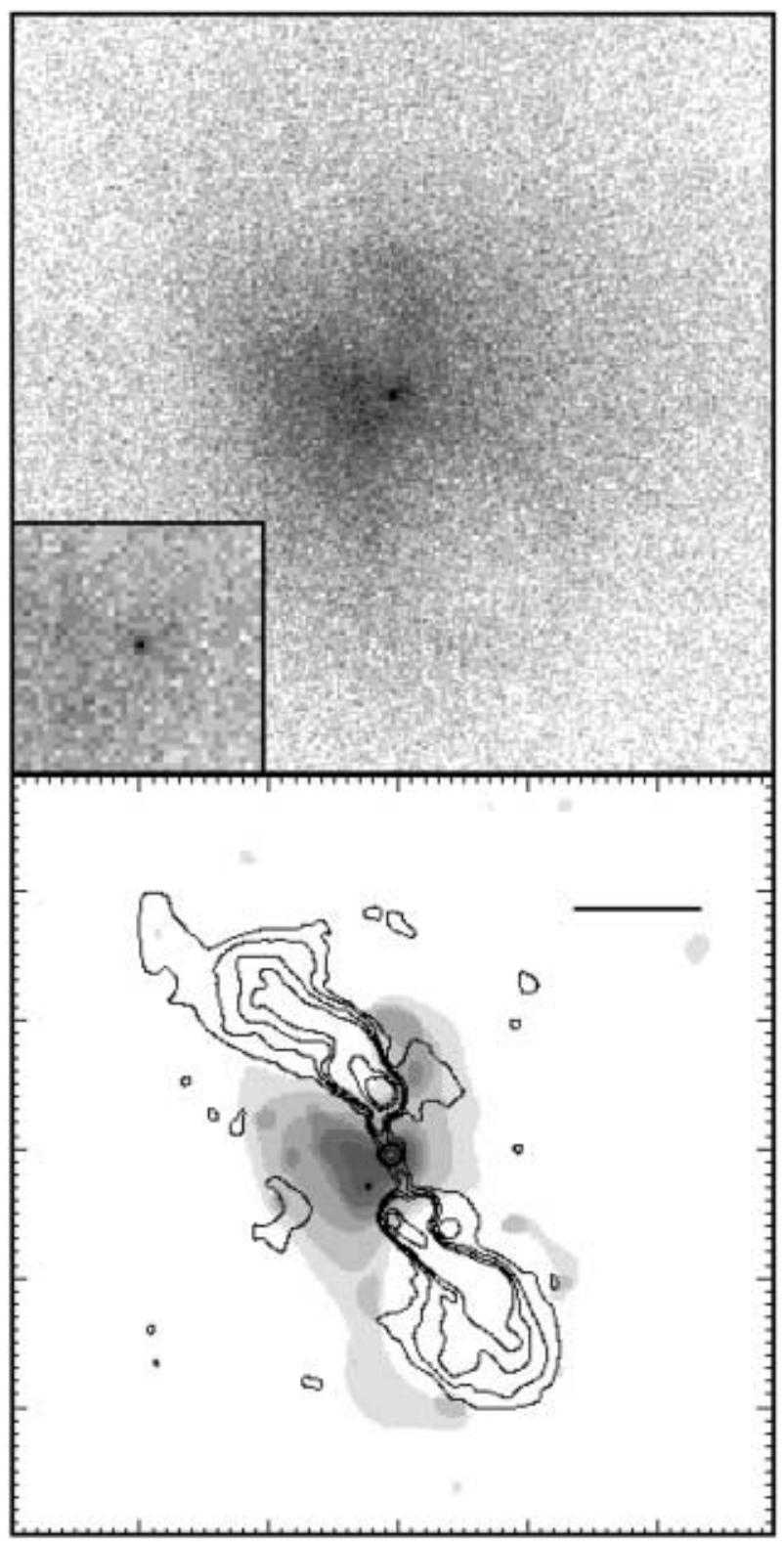

Figure 8 Upper panel: Combined Chandra ACIS-I and ACIS-S $\mathrm{X}$-ray images of the central region of the Hydra A Cluster, centred on the nuclear point source (inset). Lower panel: Wavelet smoothed image of the same region together with contours from a $6 \mathrm{~cm}$ VLA radio image of Hydra A. The scale bar is $20^{\prime \prime}$ long. See McNamara et al. (2000) for details. (P. Nulsen)

Although much of the ICM gas in clusters is presumed to have been 'left over' in some way from the process of galaxy formation, the gas is far from primordial as it is strongly enriched with heavy elements. Indeed, it can be argued that the ICM contains a greater total mass of metals than the cluster galaxies themselves (for a recent review see Renzini 2000). Galactic winds driven by supernovae have clearly played a major role in this enrichment of the ICM, but many of the details are still unclear, even to the extent of which type of supernovae dominate the process (e.g. Gibson, Loewenstein, \& Mushotzky 1997). However a strong case can be made, based on iron abundances, that metals in the ICM come from massive stars in the same 


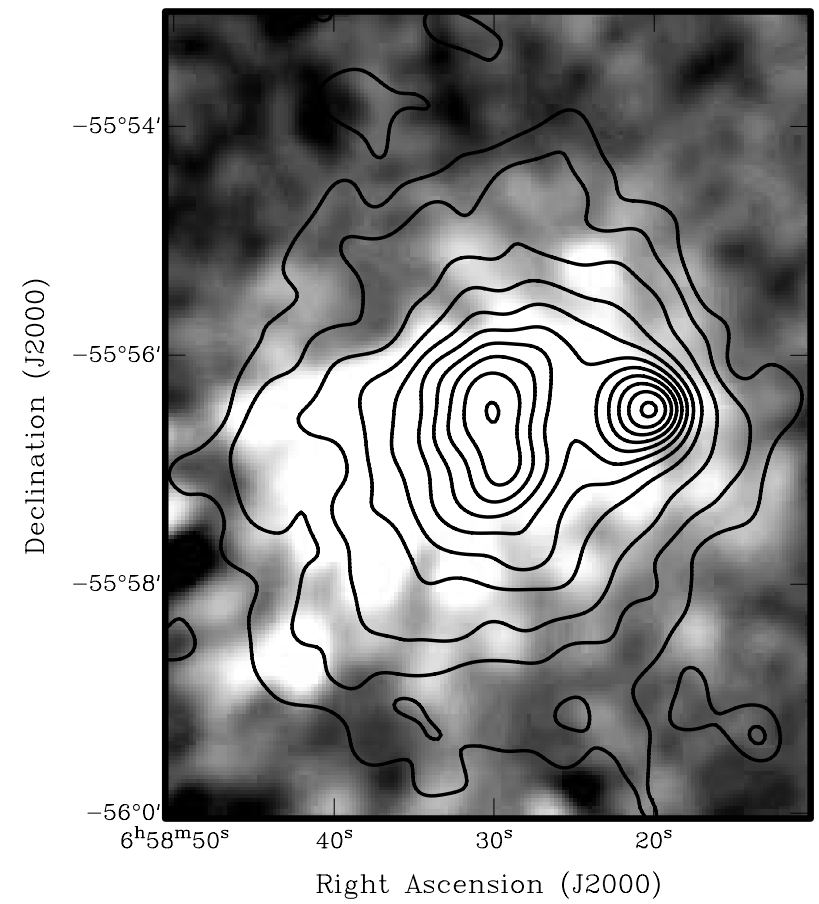

Figure 9 The powerful radio halo in the cluster 1E0657-56. The greyscale shows the radio halo measured at $1.3 \mathrm{GHz}$ with the ATCA after the removal of individual sources. The overlaid contours show the Rosat X-ray image of the cluster. See Liang et al. (2000) for details. (H. Liang)

populations as most of the light in clusters today (Gibson, this workshop).

\subsection{High Energy Particles and Magnetic Fields}

Several X-ray luminous clusters also have diffuse, steep spectrum radio emission known as 'radio halos'. The steep spectra imply the source of the radiation is synchrotron emission, but the origin of the magnetic fields and high energy particles is unclear (see Feretti \& Giovannini 1996 for a review). A promising mechanism to produce the magnetic fields and the high energy particles is by cluster mergers ${ }^{13}$. However there is a strong correlation between radio and X-ray luminosities in the clusters with radio halos, and Liang et al. (2000) show that the radio emission has a similar distribution to the X-ray emission in the cluster 1E0657-56 (see Figure 9). They propose that electrons from the very high energy tail of the thermal gas distribution provide the source of relativistic particles for the radio halo synchrotron emission.

The magnetic fields in clusters can be measured (almost) directly through the Faraday rotation of background radio sources by the cluster magnetic field. Although these measurements are difficult, the results from many clusters can be combined to show clear detection of the cluster magnetic field out to a projected radius of $800 \mathrm{kpc}$ from the cluster centres ${ }^{14}$. In a recent study at

\footnotetext{
13 Tribble (1993).

${ }^{14}$ Clarke, Kronberg, \& Böhringer (2001).
}

smaller projected radii Johnston-Hollitt et al. (this workshop) find rotation measures of $200 \mathrm{rad} \mathrm{m}^{-2}, 75-150 \mathrm{kpc}$ from the cluster centre; see Figure 10. These observations measure the rotation measure directly; the inferred magnetic field strength is model-dependent (rotation measure depends on the parallel field component) and corresponds to $0.5-3 \mu \mathrm{G}$ for a simple 'slab' model and $5 \mu \mathrm{G}$ for 'tangled' models.

The extreme case of diffuse cluster radio emission is that of 'relic' radio sources at the edges of clusters. These are extremely large (Mpc scale) diffuse sources without optical counterparts and are only found in clusters with a lot of X-ray gas. A striking example is the double relic structure imaged by the ATCA in the rich southern cluster Abell 3667 (Johnston-Hollitt, Ekers, \& Hunstead 2001) as shown in Figure 11. The origin of this relic has been modelled as resulting from shocks in the ICM caused by cluster merging ${ }^{15}$. The merger picture for Abell 3667 is supported by its bimodal distribution of galaxy velocities. High resolution very large array (VLA) radio imaging of other relic sources have revealed them to consist of very highly structured filaments (Slee et al. 2001). The filaments appear to be in pressure equilibrium with the surrounding gas which inhibits their expansion and cooling. The extreme size of the Abell 3667 relic is emphasised in Figure 11 by the inclusion of the VLA image of the relic in Abell 85 on the same scale. It should be noted however that a counterpart to the merger picture has been found in the poor southern cluster ACO S753 which contains a prominent halo feature on one side of the cluster, although there is no evidence for any merger activity (Subrahmanyan, this workshop).

Moving to higher energies, there is good reason to believe that the cluster ISM contains a significant flux of cosmic rays that have escaped from the galaxies. The energy distribution of Galactic cosmic rays extends to such high levels (already known to exceed $10^{18} \mathrm{eV}$ ) that the most energetic have radii of gyration in Galactic magnetic fields greater than a few kpc so they will escape into the Local Group (Clay 2000, 2001). We should therefore expect to find high energy cosmic rays in clusters if the galactic and cluster magnetic fields can force them to diffuse out of galaxies into the ICM. If there is any significant amount of hydrogen present in the ICM (a number density greater than about $3 \times 10^{-3} \mathrm{~cm}^{-3}$ ), these should result in a detectable flux of gamma rays (Clay, this workshop).

\section{Closing}

In this workshop, the breadth of Australian research on galaxy clusters was demonstrated by presentations discussing observations ranging from the radio through to gamma rays and cosmic rays. The Australian national observatories are continuing to develop new, worldleading facilities, so we can only expect this abundance of high quality data to continue. The challenge, as always,

${ }^{15}$ Roettiger, Burns, \& Stone (1999). 


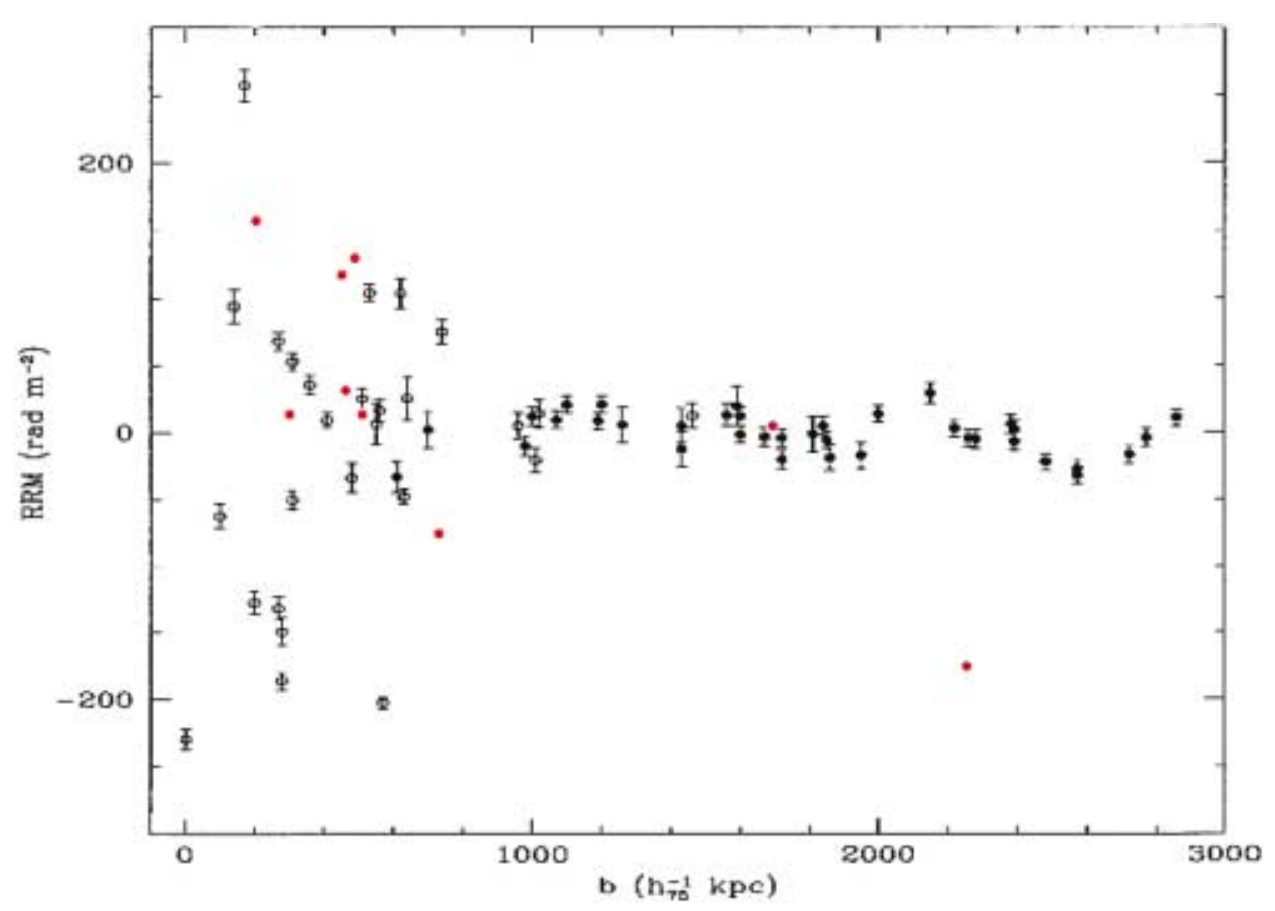

Figure 10 The detection of magnetic fields in clusters: rotation measure plotted against projected distance from cluster cores. The black points are from Clarke et al. (2001) and represent Faraday rotation measure (FRM) fits for sources in and behind a sample of northern non-cooling flow clusters; the red points are FRM fits for data taken at four radio frequencies on the ATCA for sources in and behind the cores (within $800 \mathrm{kpc}$ ) of a sample of seven southern non-cooling flow clusters (Johnston-Hollitt et al., this workshop). (M. Johnston-Hollitt)

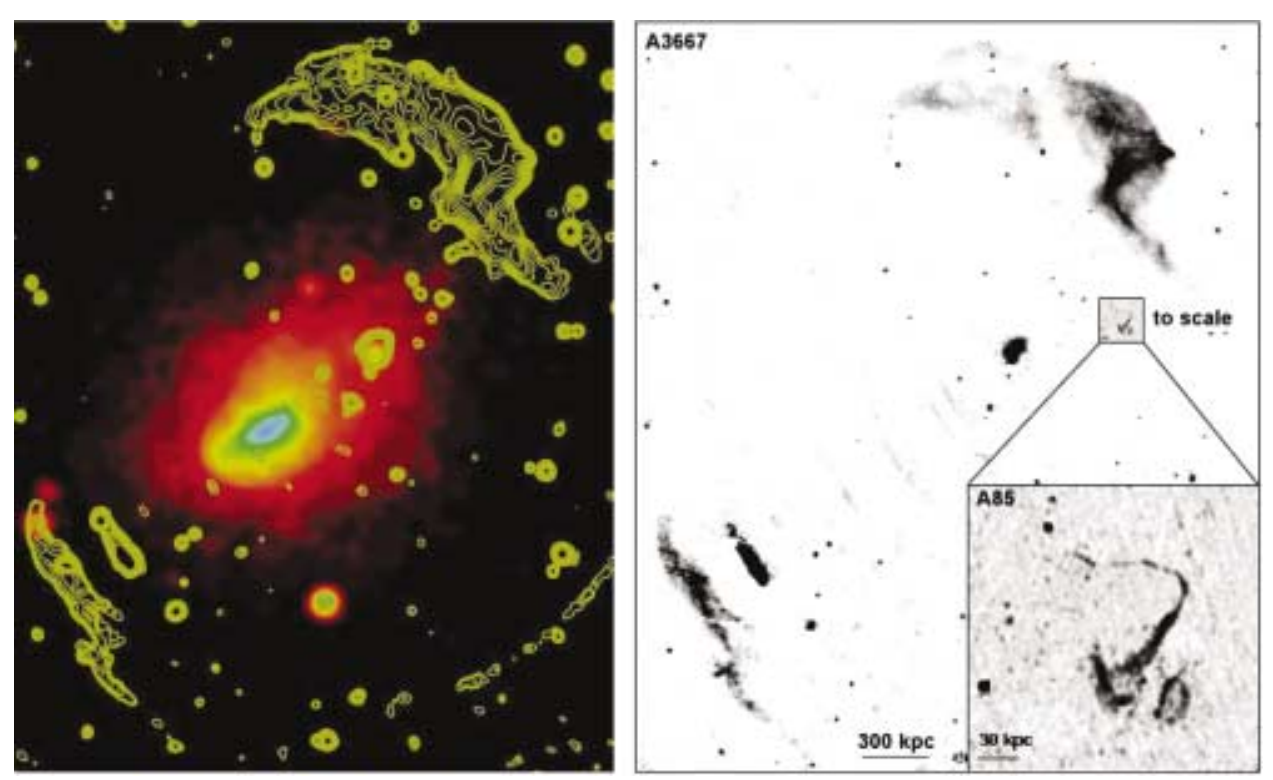

Figure 11 The giant radio relic sources in Abell 3667. Left: Rosat X-ray image of the cluster overlaid with contours of ATCA $1.4 \mathrm{GHz}$ radio image. Right: The same ATCA radio image (greyscale) with an inset showing the (Slee et al. 2001) VLA image of the relic in Abell 85 overlaid to scale. This illustrates the different scale sizes of 'relic' sources. (M. Johnston-Hollitt \& O. B. Slee)

in our small community will be to exploit these data fully by using them to develop new physical understanding. This will come about in two ways: first by bringing experts from the different observational domains together to share and discuss the data, as took place at this workshop. The relatively small size of the Australian community can be an advantage here, facilitating more interaction between observers. Secondly we need a strong theoretical effort to synthesise these results and develop new theory. The workshop also provided examples of this with numerical work presented by several speakers. Further development of the theoretical background should be a priority for us.

Finally, in our use of galaxy clusters to study galaxies, we should not forget the 'chicken and egg' problem: do galaxies drive changes in the ICM or is it the other way around? This question was posed by Ekers at the 
workshop. Although our discussions of galaxy evolution are focused on the effect the cluster environment has on galaxies, it clearly works the other way too. The X-ray gas in particular has been considerably enriched from primordial conditions given the amount of iron emission. A further example is the effect of radio galaxies on the X-ray emission at the centres of clusters. We need to take note of the feedback processes going on between cluster galaxies and their environment.

\section{Acknowledgments}

On behalf of all the participants at the workshop I would like to thank Warrick Couch, Ron Ekers, and Melanie Johnston-Hollitt for organising the meeting. We also thank the Australia Telescope National Facility for financial support. I particularly wish to thank all the participants who provided me with material for this review and Andrew Melatos and Paul Nulsen in particular for their comments on an early draft of the paper.

\section{References}

Abell, G. O. 1958, ApJS, 3, 211

Abell, G. O., Corwin, H. G., \& Olowin, R. P. 1989, ApJS, 70, 1 (ACO)

Bahcall, N. A., Cen, R., Davé, R., Ostriker, J. P., \& Yu, Q. 2000, ApJ, 541,1

Barnes, D. G., et al. 2001, MNRAS, 322, 486

Bekki, K., Couch, W. J., \& Drinkwater, M. J. 2001a, ApJ, 522, L105

Bekki, K., Shioya, Y., \& Couch, W. J. 2001b, ApJ, 547, L17

Binney, J., \& Tremaine, S. 1987, Galactic Dynamics (Princeton, NJ: Princeton University Press)

Binggeli, B., Sandage, A., \& Tammann, G. A. 1988, ARA\&A, 26, 509

Boyce, P. J., Phillipps, S., Jones, J. B., Driver, S. P., Smith, R. M., \& Couch, W. J. 2001, MNRAS, 328, 277

Burns, J. O. 1998, Science, 280, 400

Butcher, H., \& Oemler, A. 1978, ApJ, 219, 18

Carlberg, R. 2001, in Encyclopedia of Astronomy and Astrophysics, ed. Paul Murdin (Basingstoke: Nature Publishing Group), 880

Carlstrom, J. E., Joy, M., Holder, G., Holzapfel, W. L., Laroque, S., Mohr, J. J., \& Reese, E. 2000, in Constructing the Universe with Clusters of Galaxies, IAP 2000 Meeting, Paris, France, July 2000, eds Florence Durret, \& Daniel Gerbal, available on CD-Rom and at http://www.iap.fr/Conferences/Colloque/ coll2000/contributions, Ref.4.4.1

Clarke, T. E., Kronberg, P. P., \& Böhringer, H. 2001, ApJ, 547, L111

Clay, R. W. 2000, PASA, 17, 212

Clay, R. W. 2001, PASA, 18, 148

Clowe, D., \& Schneider, P. 2001, A\&A, 379, 384

Colless, M. M., \& Dunn, A. M. 1996, ApJ, 458, 435

Colless, M. M., et al. 2001, MNRAS, 328, 1039

Conselice, C. J., Gallagher, J. S., \& Wyse, R. F. G. 2001, ApJ, 559, 791

Couch, W. J., \& Sharples, R. M. 1987, MNRAS, 229, 423

Couch, W. J., Ellis, R. S., Godwin, J., \& Carter, D. 1983, MNRAS, 205,1287

Couch, W. J., Barger, A. J., Smail, I., Ellis, R. S., \& Sharples, R. M. 1998, ApJ, 497, 188

Couch, W. J., Balogh, M. L., Bower, R. G., Smail, I., Glazebrook, K., \& Taylor, M. 2001, ApJ, 549, 820

David, L. P., Nulsen, P. E. J., McNamara, B. R., Forman, W., Jones, C., Ponman, T., Robertson, B., \& Wise, M. 2001, ApJ, 557, 546
Dekel, A., \& Silk, J. 1986, ApJ, 303, 39

de Vaucouleurs, G., de Vaucouleurs, A., Corwin, J. R., Buta, R. J., Paturel, G., \& Fouque, P. 1991, Third Reference Catalogue of Bright Galaxies (New York: Springer-Verlag), 18

Diaferio, A. 1999, MNRAS, 309, 610

Dressler, A. 1980, ApJ, 236, 351

Dressler, A. 1984, ARA\&A, 22, 185

Drinkwater, M. J., \& Gregg, M. D. 1998, MNRAS, 296, L15

Drinkwater, M. J., Gregg, M. D., \& Colless, M. 2001a, ApJ, 548, L139

Drinkwater, M. J., Gregg, M. D., Holman, B. A., \& Brown, M. J. I. 2001b, MNRAS, 326, 1076

Drinkwater, M. J., Jones, J. B., Gregg, M. D., \& Phillipps, S. 2000b, PASA, 17, 227

Drinkwater, M. J., et al. 2000a, A\&A, 355, 900

Durret, F., \& Gerbal, D. 2000, Constructing the Universe with Clusters of Galaxies, IAP 2000 Meeting, Paris, France, July 2000, eds Florence Durret, \& Daniel Gerbal, available on CD-Rom and at http://www.iap.fr/Conferences/Colloque/coll2000/contributions

Ebeling, H., Edge, A. C., Allen, S. W., Crawford, C. S., Fabian, A. C., \& Huchra, J. P. 2000, MNRAS, 318, 333

Edwards, S., \& Colless, M., 2001, AAO Newsletter, 98, 9

Ettori, S., \& Fabian, A. C. 1999, MNRAS, 305, 834

Fabian, A. C. 1994, ARA\&A, 32, 277

Feretti, L., \& Giovannini, G. 1996, in Extragalactic Radio Sources, IAU Symposium 175, eds Ron D. Ekers, C. Fanti, \& L. Padrielli (Dordrecht: Kluwer Academic Publishers), 333

Ferguson, H. C. 1989, AJ, 98, 367

Ferguson, H. C., \& Sandage, A. 1988, AJ, 96, 1520

Francis, P. J., Wilson, G. M., Woodgate, B. E. 2001, PASA, 18, 64

Gibson, B. K., Loewenstein, M., \& Mushotzky, R. F. 1997, MNRAS, 290,623

Ikebe, Y., et al. 1996, Nature, 379, 427

Johnston-Hollitt, M., Ekers, R. D., \& Hunstead, R. W. 2001, in Particles and Fields in Radio Galaxies: Proceedings of the Oxford Radio Galaxy Workshop, Oxford, 3-5 August 2000, eds R. Bundell, \& K. Laing, in press

Klamer, I. 2000, Senior Physics project, University of Sydney

Kneib, J.-P., Ellis, R. S., Smail, I., Couch, W. J., \& Sharples, R. M. 1996, ApJ, 471, 643

Lewis, I. J., et al. 2001, MNRAS, submitted

Liang, H., Hunstead, R. W., Birkinshaw, M., \& Andreani, P. 2000, ApJ, 544, 686

Lo, K. Y., Chiueh, T., Liang, H., Ma, C. P., Martin, R., Ng, K.-W., Pen, U. L., \& Subramanyan, R. 2000, IAU Symposium, 201, 31

Machacek, M. E., Bautz, M. W., Canizares, C. R., \& Garmire, G. P. 2001, ApJ, submitted, astro-ph/0109263

Markevitch, M., et al. 2000, ApJ, 541, 542

McNamara, B. R., et al. 2000, ApJ, 534, L135

Poggianti, B. M., Smail, I., Dressler, A., Couch, W. J., Barger, A. J., Butcher, H., Ellis, R. S., \& Oemler, A. J. 1999, ApJ, 518, 576

Poggianti, B. M., et al. 2001, ApJ, 563, 118

Praton, E. A., \& Schneider, S. E. 1994, ApJ, 422, 46

Read, A. M., Filipovic, M. D., Pietsch, W., \& Jones, P. A. 2001, A\&A, 369, 467

Renzini, A. 2000, in Large Scale Structure in the X-ray Universe, Proceedings of the 20-22 September 1999 Workshop, Santorini, Greece, eds M. Plionis, \& I. Georgantopoulos (Paris, France: Atlantisciences), 103

Roettiger, K., Burns, J. O., \& Stone, J. M. 1999, ApJ, 518, 603

Saunders, W., et al. 2001, AAO Newsletter, 97, 14

Schindler, S. 2001, in Multifrequency Behaviour of High Energy Cosmic Sources, ed. F. Giovannelli, in press, astro-ph/0109040

Schröder, A., Drinkwater, M. J., \& Richter, O.-G. 2001, A\&A, 376,98

Slee, O. B., Roy, A. L., Murgia, M., Andernach, H., \& Ehle, M. 2001, AJ, 122, 1172

Smail, I., Edge, A. C., Ellis, R. S., \& Blandford, R. D. 1998, MNRAS, 293, 124 
Sunyaev, R. A., \& Zel'dovich, Ya. B. 1972, Comm. Astrophys. Sp. Phys., 4, 173

Tremaine, S. 1992, Physics Today, 45, 28

Tresse, L., \& Maddox, S. J. 1998, ApJ, 495, 691

Tribble, P. C. 1993, MNRAS, 263, 31

Tully, R. B., Somerville, R. S., Trentham, N., \& Verheijen, M. A. W. 2001, ApJ, submitted, astro-ph/0107538

Tyson, J. A. 1996, in Astrophysical Applications of Gravitational Lensing: Proceedings of the 173rd Symposium of the International Astronomical Union, eds C. S. Kochanek, \& Jacqueline N. Hewitt (Dordrecht: Kluwer Academic Publishers), 107

van Gorkom, J. 1996, in The Minnesota Lectures on Extragalactic Neutral Hydrogen, ASP Conference Series 106, ed. E. D. Skillman (San Francisco: ASP), 293
Waugh, M., Webster, R. L., \& Drinkwater, M. J. 2000, in Mapping the Hidden Universe, ASP Conference Series 218, eds R. C. Kraan-Korweg, P. A. Henning, \& H. Andernach (San Francisco: ASP), 231

White, S. D. M., \& Frenk, C. S. 1991, ApJ, 379, 52

Wu, K. K. S., Fabian, A. C., \& Nulsen, P. E. J. 2000, MNRAS, 318,889

Zabludoff, A. I., \& Zaritsky, D. 1995, ApJ, 447, L21

Zwaan, M. A., van Dokkum, P. G., \& Verheijen, M. A. W. 2001, Science, 293, 1800

Zwicky, F. 1937, ApJ, 86, 217 